\title{
Public Health Risk Assessment Model (PH-RAM) for Internally Displaced Persons (IDP) camps - A case study of North Darfur, Sudan
}

Elfadil Mohammed Mahmoud ( $\sim$ elfadil74@gmail.com )

Asian Institute of Technology https://orcid.org/0000-0003-3871-3797

Indrajit Pal

Asian Institute of Technology

Haitham Bashier Abbas

World Health Organization Regional Office For the Eastern Mediterranean

\section{Research}

Keywords: Health hazard, Vulnerability, Public health risk, Assessment, IDPs, North Darfur, Sudan.

Posted Date: September 9th, 2020

DOI: https://doi.org/10.21203/rs.3.rs-71573/v1

License: (c) (i) This work is licensed under a Creative Commons Attribution 4.0 International License.

Read Full License 


\section{Abstract}

Background: Internally Displaced Persons (IDPs) in North Darfur of Sudan have been experiencing serious public health risks such as high prevalence rate, and outbreaks of communicable diseases. Public health risk assessment is complex as researchers engaged in disaster science and management use different quantitative models with different interpretations. The objective of the present study is to proposes methodology for developing Public Health Risk Assessment Model (PH-RAM) to quantify and conceptualizing public health risk as a function of hazard, vulnerability, and capacity.

Methods: Household-level public health risk has been assessed integrating the three main component indices, hazard, vulnerability, and capacity, using primary data collected from households of the three IDP camps. Administered survey was performed in 326 households in three IDP camps (Shangil Tobaya, Zamzam, and Abu Shouk) using cluster sampling technique. Households were classified into four public health risk levels, i.e., low risk, moderate risk, high risk, and very high risk.

Results: The result shows that the levels of public health risk varied significantly across households in three camps. Households of the Shangil Tobaya camp (in a rural area) were found to be the most vulnerable and the most at high risk to communicable diseases compared to other two camps because of insufficient health services, inadequate water, poor sanitation, and low level of income. The study recommended, mitigation and prevention measures to reduce the factors that are increased the vulnerability of the IDPs to health risks.

Conclusions: Our study shows that the suggested PH-RAM could be served as household-level public health risk assessment tools in the context of IDPs at the local, national and regional levels.

\section{Background}

Armed conflict often causes massive displacement of people due to insecure situations, destruction of homes, and their livelihoods (1). By the last of 2018, there were an estimated over 40 million persons as internally displaced persons within the borders of 56 countries as a result of armed conflict (2). Sudan has suffered from years of civil conflicts since1956. In Sudan, 2.3 million people were displaced by armed conflict (3). North Darfur State is one of the most affected states in Darfur region, western Sudan. Long-standing droughts, internal conflicts, and political instability led Darfur region to widespread of armed conflict in February 2003, which killed 298,271 persons (4). Nearly two million persons had been displaced to camps due to the peak period of conflict in 2003 and 2004. Figure 1 describes the number of IDPs displaced by armed conflict per year in Darfur from 2003-2017 (3)

Currently, IDPs in Darfur were exposed to prolonged displacement in temporary camps characterized by overcrowding and poor living conditions, which make them vulnerable to several types of health hazards such as the epidemic of communicable diseases (5). Further, their vulnerability to such health risks was 
increased due to lack of access to adequate water, inadequate latrines, solid waste collection, and primary healthcare services (5). In addition, the humanitarian partners in the field also facing lack of funding from the donors because of protracted Darfur crisis. All these factors directly increased the vulnerability of IDPs to health risks and promote communicable diseases outbreaks (i.e. diarrhea, malaria, and measles). Like most of the developing countries, Sudan is lacking clear and comprehensive public health risk assessment tools and methods in the context of IDPs. A clear understanding of explaining public health risk through the lens of disaster risk components and providing quantitative tools for risk assessment is a big gap. Constructing public health risk indexes through the integrated hazard, vulnerability and capacity analyses is also one the major gaps (6). Our objective is to proposes a Public Health Risk Assessment Model (PH-RAM) by integrating the three main component indexes i.e., health hazard index, vulnerability, and capacity index that aims to assess the public health risk of the (IDPs) in three camps (Shangil Tobaya, Zamzam and Abu Shouk) in North Darfur region in Sudan. PHRAM will be useful as a tool for assessing public health risk of the IDPs and suggesting appropriate public health risk reduction strategies in the context of IDPs. Most of the available approaches follow rapid assessment of public health risk which focuses on ad-hoc collation of all available evidences and information about the health problem in order to make an urgent intervention to control the public health risk. No single research goes further to find the factors that raised the vulnerability of affected community to such health risk and their capacity to cope. Thus, developing a new method that allows assessing public health risk integrating hazard, vulnerability and capacity are paramount for the public health risk reduction measures and strategies.

Nowadays, wars and natural hazards pushed a large number of people to move from their original living areas and settle in temporary camps with various challenges on access to safe water, adequate sanitation and, lack of access to health services, etc.(7). All these factors were increased the health hazard and vulnerability of the affected population to public health risks, and often claim more lives of the IDPs than the conflict itself (8). In recent years, health has been recognized explicitly as both, a determinant and an outcome of the human dimension of disaster risk reduction (6). Risk measurement is complex as researchers involved in disaster science and management use various quantitative models (9). Public health risk can be conceptualized as a function of the health hazard, vulnerability, and capacity (10). The study explains hazard, vulnerability, and capacity through the lens of public health risk.

Hazard is defined as "a dangerous phenomenon that may cause negative health impacts" (11). Displacement can lead and exposed the IDPs to several types of public health hazards, but the common one is an epidemic of communicable diseases (12). An epidemic disease is defined as "the number of cases of disease increases above what would normally be expected in a defined community, geographical area or season" (13). Health hazards only convert into epidemic diseases due to the presence of vulnerable population and low-level capacity as preparedness and mitigation measures to cope with health hazards (12). Globally, diarrhea, malaria, and measles are the major communicable diseases caused the high morbidity and mortality among the IDPs and refugees in camp settings (14). Present research in IDP camps in Sudan considers the physical public health conditions as indicators to 
assess hazard (e.g., frequency and severity of the epidemic diseases in IDP camps at the household level).

The term vulnerability has been used in different disciplines such as health, disaster risk reduction and climate change (15). In the context of disaster risk reduction, vulnerability is defined as the likelihood of suffering from adverse health effects when exposed to a given health hazard (16). In this study, vulnerability refers to economic, social, health, and environmental health factors that make people susceptible to the impacts of conflict and displacement and their adverse health consequences. People displaced by natural or man-made hazard their vulnerabilities to public health risks have been raised due to several factors (17). First, IDPs receiving inadequate health services in terms of inaccessibility, high cost, or lack of supplies (14). Second, IDP experience a lack of access to safe drinking water, poor sanitation, the absence of a solid waste management system, and poor hygiene practices (5) Third, a lack of financial capacity prevents the affected population to response to their needs (18).

Capacity in the light of disaster risk science can be defined as "ability of people, organizations and systems, using available skills and resources, to face and manage adverse conditions, emergencies or disasters" (11). Capacity refers to the ability to react to and reduce the adverse effects of potential or experienced hazards. It includes both current community capacity to cope with the public health hazards (epidemic diseases) in camps and future action to improve the ability to prepare (19). Capacity adaptive to public health risks depends on several factors such as economic wealth, knowledge about disease transmission and prevention, institutions, availability of basic services, community engagement, and participation (20). Preparedness activities in humanitarian settings greatly increase the community's resilience and capacity $[(21 ; 22 ; 23]$

The term risk is understood in different ways by different disciplines. According to UNISDR, the risk is defined as the harmful consequences resulting from interactions between hazards, exposure, and vulnerable conditions (11). While the public health risk refers to "the probability of disease developing in an individual in a specified time interval" (14), Poor of knowledge and technical training, lack of access to information about hazards, and a low level of perceived risk fuel unsafe health behaviors among households of IDP camps (23). Protracted conflicts and subsequent displacements were exposed IDPs to several types of health hazard with greater vulnerability, and low capacity made them at high risk to communicable diseases (5). Based on the literature review, we can conclude that the relationship of the health hazard, vulnerability, and capacity with public health risk is conceptualized with the context of disaster risk. Risk is directly related to the product of health hazard and vulnerability and inversely related to coping/adaptive capacity $[10 ; 24]$

Risk assessment can help in identifying risks, vulnerabilities and also helps to improve capacity building of society (25). Various definitions, approaches, and quantitative methods are used for risk assessment in the context of disaster risk science and climate change literature [26; 27; 28]. Public health risk assessment is the process of estimating the probability of adverse health effects in humans who may be exposed to different hazards (10). Currently, there is a big gap in assessing public health risk through 
disaster risk context. For example, World Health Organization (WHO) and other researchers are more focused on rapid risk assessment approach for assessing public health risk and primarily focused on rapid collection of ad-hoc information about the public health event for quick response (29), and did not go further to find the factors that increase the vulnerability of affected community to such risk and their capacity to cope. Thus, developing a new method that allows assessing public health risk integrating hazard, vulnerability and capacity are paramount for the public health risk reduction measures and strategies. In this regard, we followed the disaster risk concept to develop a public health risk index. A composite index method was used to combine three main components (hazard index, vulnerability index, and capacity index) as primary functions of public health risk (phR).

\section{Risk $(\mathrm{phR})=\frac{\text { Hazard }(\mathrm{phH}) \times \text { Vulnerability }(\mathrm{phV})}{\text { Capacity }(\mathrm{phC})}$}

Nowadays, there is a lack of a common public health risk assessment framework (30). Filed visit to the IDP camps emphasized that the IDP camps with multifaceted challenges especially with the basic services The present research conceptualize the public health risks considering three fundamental components namely public health hazards $(\mathrm{phH})$, public health vulnerability $(\mathrm{phV})$ and public health capacity (phC). The following components were considered in the conceptual framework for public health risk index at the household level (Fig. 2). The first component is a public health hazard assessed through the frequency and severity of epidemics of communicable diseases. The second component is public health vulnerability assessed through four vulnerability components such as (1) socio-economic (i.e. gender, age, education, employment, income, and family size); (2) access to environmental health services (i.e. water, sanitation, solid waste management, dispose of the child feces, washing soap, hygiene kits), (3) and access to primary health services (i.e. vaccination, antenatal care services, walking time to health centers, mosquito bed net utilization). The third component is capacity assessed through three fundamental factors (i.e. preparedness planning, saving and multi-economic options, and awareness about diseases prevention and control). Primary data were collected through questionnaires from households of three IDPs camps in February and March 2018.

\section{Methods}

\section{Study area}

The study was conducted in North Darfur state which was one of the most affected states during the Darfur conflict in 2003 . The state covers 296,420 sq.-km equivalent to $12 \%$ of the area of Sudan (31). The study area was significantly affected by the armed conflict compared to other states in Sudan and gradually accommodated about 576,227 persons in different IDP camps (32). Three IDP camps namely, Zamzam, Abu Shouk, and Shangil Tobaya has been selected for the present research, based on certain predefined criteria for example the camp with low service coverage and poor health indicators, etc (Fig. 3). 


\section{Sampling And Data Collection}

A total of 36,866 households lived in three selected camps in Zamzam $(25,525)$, Abu Shouk $(7,880)$, and Shangil Tobaya $(3,461)(32)$. (33). Yamane (1967) formula was used to calculate the sample size 202.95 with the level of precision, set as (0.07). The design effect was chosen as 1.5 and calculated because of cluster sampling (34). Additional 7\% sample size also included to cover the non-responsive households and finally, the total number of households surveyed was 326 . The actual number of respondents per camp was calculated through the propositional sampling for Zamzam, Abu Shock, and Shangil Tobaya IDP camps 225,68 , and 33 , respectively.

$$
\mathrm{n}=\frac{\mathrm{N}}{1+\mathrm{N}(\mathrm{e})^{2}}
$$

Where, $\mathrm{n}=$ Sample size, $\mathrm{N}=$ total numbers of household in all targeted camps $(36,866)$ $\mathrm{e}=$ level of precision, set as (0.07).

\section{Data Sources And Collection Procedures}

The survey was conducted in 2018 to collect primary and secondary data from three IDP camps and State Ministry of Health and International humanitarian partners. A structured questionnaire was used to collect the primary data. Questionnaire was translated into Arabic language for easy understanding. Pretesting of the questionnaire was also conducted to ensure the accuracy of the translation as well as relevancy of the questions to the local context. Eight health officers ( 4 male and 4 female) were contracted from the State Ministry of Health, to assist the researcher in household data collection. Informed consent was taken from head of the household before the interview. The two-stage cluster sampling method was used to collect primary data from households. In the first stage, a total number of 30 clusters were allocated to the three targeted IDP camps proportionate to their sample size of 326 households. Due to a large number of households in the camps, a second stage cluster was used to randomly select zones within the sampled IDP camps to serve as clusters, and the number of households per zone was identified, then households were selected based on the modified (WHO) Expanded Program of Immunization cluster sampling technique. Finally, a total of 326 respondents (Zamzam 225, Abu Shouk 68, and Shangil Tobaya 33), were interviewed in three camps.

\section{Selection of indicators and weights for hazard, vulnerability, and capacity}

An extensive literature review was conducted to select the relevant indicators (Table 1) to develop public health hazard index $(\mathrm{phHI})$, public health vulnerability index $(\mathrm{phVI})$ and public health capacity index (phCl). Two indicators were used for the phHI, twenty-two indicators were used for the phVI and ten indicators were used for $\mathrm{phCl}$ to define public health risk index (phRI). Each indicator was classified into 
classes for example, gender classified into male and female, other indicators classified to more than two and three classes as needed. Weight was assigned to each class within the indicator, based on the level of vulnerability, capacity, or hazard. Each indicator had a maximum weight of 1 . The highest vulnerable classes were assigned with the weight value as 1 , while the lowest vulnerable as 0 . After computing, the composite vulnerability index (CVIs), for health vulnerabilities, the CVIs are transformed to $0-1$ scale. The indicators with three classes were assigned the values $0.33,0.67$, and 1 ; for four classes, the values were $0.25,0.50,0.75$, and 1 ; and for five classes, the values were $0.2,0.4,0.6,0.8$, and 1 . Thus, the composite index for each component fell between 0 and 1 . 
Table 1

Indicators and transformed values for health hazard, vulnerability and capacity at household level in IDPs camp in North Darfur/Sudan

$\begin{array}{lllll}\text { S. Indicators } & \text { classes } & \begin{array}{l}\text { Weighing } \\ \text { scale }\end{array} & \text { Interpretation } & \begin{array}{l}\text { Selected } \\ \text { indicators } \\ \text { used by } \\ \text { other } \\ \text { studies }\end{array} \\ & & & & \begin{array}{c}\text { stion } \\ \end{array} \\ & & & \end{array}$

1. public health Hazard indicators $(\mathrm{phH})$

\begin{tabular}{|c|c|c|c|c|c|}
\hline \multirow[t]{5}{*}{1} & & 0 & 0 & \multirow{5}{*}{$\begin{array}{l}\text { The past of diseases } \\
\text { outbreaks shows the camp } \\
\text { is at high to public health } \\
\text { risks }\end{array}$} & \multirow[t]{5}{*}{ (38) } \\
\hline & \multirow{4}{*}{$\begin{array}{l}\text { epidemic diseases in } \\
\text { the last five years }\end{array}$} & $1-2$ times & 0.25 & & \\
\hline & & $3-4$ times & 0.5 & & \\
\hline & & $5-6$ times & 0.75 & & \\
\hline & & $>6$ times & 1 & & \\
\hline \multirow[t]{5}{*}{2} & \multirow{5}{*}{$\begin{array}{l}\text { The severity of the } \\
\text { epidemic diseases in } \\
\text { the last five years at } \\
\text { household in terms of } \\
\text { people affected }\end{array}$} & 0 & 0 & \multirow{5}{*}{$\begin{array}{l}\text { A high number of people } \\
\text { affected by diarrhea and } \\
\text { acute respiratory infection } \\
\text { (ARI) at the household } \\
\text { level, meaning that more } \\
\text { severity and result in high } \\
\text { morbidity and mortality. }\end{array}$} & \multirow[t]{5}{*}{ (38) } \\
\hline & & $1-2$ & 0.25 & & \\
\hline & & $3-4$ & 0.50 & & \\
\hline & & $5-6$ & 0.75 & & \\
\hline & & $>6$ & 1 & & \\
\hline
\end{tabular}

\section{2. public health Vulnerability indicators (phV)}

\begin{tabular}{|c|c|c|c|c|c|}
\hline \multirow[t]{2}{*}{1} & \multirow[t]{2}{*}{ Gender } & Female & 1 & \multirow{2}{*}{$\begin{array}{l}\text { Female-headed household } \\
\text { is more vulnerable }\end{array}$} & \multirow[t]{2}{*}{ (36) } \\
\hline & & Male & 0.5 & & \\
\hline \multirow[t]{5}{*}{2} & \multirow{5}{*}{$\begin{array}{l}\text { Age of head of } \\
\text { household } \\
\text { (respondents) }\end{array}$} & $18-30$ & 0.25 & \multirow{5}{*}{$\begin{array}{l}\text { elderly and children are } \\
\text { more vulnerable to health } \\
\text { hazard and risks }\end{array}$} & \multirow[t]{5}{*}{ (39) } \\
\hline & & $31-45$ & 0.50 & & \\
\hline & & $46-60$ & 0.75 & & \\
\hline & & $61-74$ & 1 & & \\
\hline & & $<18$ years & 1 & & \\
\hline \multirow[t]{5}{*}{3} & \multirow[t]{5}{*}{ Education level } & None/ Non- & 1 & \multirow{5}{*}{$\begin{array}{l}\text { Lower education level is a } \\
\text { higher level of vulnerability }\end{array}$} & \multirow[t]{5}{*}{$(40)$} \\
\hline & & curriculum & 0.75 & & \\
\hline & & Basic & 0.50 & & \\
\hline & & Secondary & 0.25 & & \\
\hline & & $\begin{array}{l}\text { University/ } \\
\text { high } \\
\text { educational }\end{array}$ & & & \\
\hline
\end{tabular}




\begin{tabular}{|c|c|c|c|c|c|}
\hline $\begin{array}{l}\text { S. } \\
\text { No }\end{array}$ & Indicators & classes & $\begin{array}{l}\text { Weighing } \\
\text { scale }\end{array}$ & Interpretation & $\begin{array}{l}\text { Selected } \\
\text { indicators } \\
\text { used by } \\
\text { other } \\
\text { studies }\end{array}$ \\
\hline \multirow[t]{3}{*}{4} & \multirow{3}{*}{$\begin{array}{l}\text { Family size } \\
\text { (members) }\end{array}$} & $01-07$ & 0.33 & \multirow{3}{*}{$\begin{array}{l}\text { A household with higher } \\
\text { members is a greater level } \\
\text { of vulnerability }\end{array}$} & \multirow[t]{3}{*}{ (41) } \\
\hline & & $08-14$ & 0.67 & & \\
\hline & & $>15$ & 1 & & \\
\hline \multirow[t]{2}{*}{5} & \multirow{2}{*}{$\begin{array}{l}\text { Numbers of earning } \\
\text { members in the } \\
\text { family }\end{array}$} & One person & 1 & \multirow{2}{*}{$\begin{array}{l}\text { Lower the number of } \\
\text { earning members higher } \\
\text { the level of vulnerability }\end{array}$} & \multirow[t]{2}{*}{ (42) } \\
\hline & & $>1$ person & 0.5 & & \\
\hline \multirow[t]{5}{*}{6} & \multirow{5}{*}{$\begin{array}{l}\text { Annual family } \\
\text { income (in Sudanese } \\
\text { pound) }\end{array}$} & $<15,000$ & 1 & \multirow{5}{*}{$\begin{array}{l}\text { Lower the income higher is } \\
\text { the level of vulnerability }\end{array}$} & \multirow[t]{5}{*}{ (43) } \\
\hline & & $15,000-$ & 0.75 & & \\
\hline & & 10,000 & 0.50 & & \\
\hline & & 21,000 & 0.25 & & \\
\hline & & $>21,000$ & & & \\
\hline \multirow[t]{8}{*}{7} & \multirow{8}{*}{$\begin{array}{l}\text { The economic } \\
\text { activity of the head of } \\
\text { household }\end{array}$} & Unemployed & 1 & \multirow{8}{*}{$\begin{array}{l}\text { The persons with a job are } \\
\text { the least vulnerable and } \\
\text { unemployed is the most } \\
\text { vulnerable }\end{array}$} & \multirow[t]{8}{*}{ (5) } \\
\hline & & student & 0.8 & & \\
\hline & & housewife & 0.8 & & \\
\hline & & Retired & 0.6 & & \\
\hline & & Self- & 0.4 & & \\
\hline & & & 0.4 & & \\
\hline & & - & 0.2 & & \\
\hline & & & & & \\
\hline \multirow[t]{3}{*}{8} & \multirow{3}{*}{$\begin{array}{l}\text { Walking time to } \\
\text { nearest health } \\
\text { facilities }\end{array}$} & $>30$ minutes & 1 & \multirow{3}{*}{$\begin{array}{l}\text { The longer walking time } \\
\text { from house to nearest } \\
\text { health facility greater the } \\
\text { vulnerability }\end{array}$} & \multirow[t]{3}{*}{ (16) } \\
\hline & & 15-30 & 0.66 & & \\
\hline & & $<15$ minutes & 0.33 & & \\
\hline \multirow[t]{3}{*}{9} & \multirow{3}{*}{$\begin{array}{l}\text { Frequency of visits to } \\
\text { antenatal care clinics } \\
\text { by pregnant women }\end{array}$} & $>9$ times & 0.33 & \multirow{3}{*}{$\begin{array}{l}\text { The health of the mother is } \\
\text { highly depending on the } \\
\text { level antenatal and } \\
\text { postnatal care received by } \\
\text { pregnant women }\end{array}$} & \multirow[t]{3}{*}{ (44) } \\
\hline & & 5 to 9 times & 0.66 & & \\
\hline & & 4 times & 1 & & \\
\hline \multirow[t]{2}{*}{10} & \multirow[t]{2}{*}{ Place of delivery } & Health & 0.5 & \multirow{2}{*}{$\begin{array}{l}\text { Women delivery at home } \\
\text { are more vulnerable to } \\
\text { risks }\end{array}$} & \multirow[t]{2}{*}{ (44) } \\
\hline & & home & 1.00 & & \\
\hline
\end{tabular}




\begin{tabular}{|c|c|c|c|c|c|}
\hline $\begin{array}{l}\text { S. } \\
\text { No }\end{array}$ & Indicators & classes & $\begin{array}{l}\text { Weighing } \\
\text { scale }\end{array}$ & Interpretation & $\begin{array}{l}\text { Selected } \\
\text { indicators } \\
\text { used by } \\
\text { other } \\
\text { studies }\end{array}$ \\
\hline \multirow[t]{2}{*}{11} & \multirow{2}{*}{$\begin{array}{l}\text { Pregnant women } \\
\text { delivered by skilled } \\
\text { birth attendants }\end{array}$} & $\begin{array}{l}\text { Skilled birth } \\
\text { attendants }\end{array}$ & 0.5 & \multirow{2}{*}{$\begin{array}{l}\text { Pregnant women delivered } \\
\text { under unskilled birth } \\
\text { attendants more vulnerable } \\
\text { to health risk }\end{array}$} & \multirow[t]{2}{*}{ (16) } \\
\hline & & $\begin{array}{l}\text { Non-skilled } \\
\text { birth } \\
\text { attendants }\end{array}$ & 1 & & \\
\hline \multirow[t]{3}{*}{12} & \multirow{3}{*}{$\begin{array}{l}\text { Children under } 24 \\
\text { months received } 2 \text { nd } \\
\text { measles vaccine }\end{array}$} & Completed & 0.33 & \multirow{3}{*}{$\begin{array}{l}\text { A household with a high } \\
\text { deficit rate of } \\
\text { immunization is very } \\
\text { highly vulnerable to risks } \\
\text { of epidemics }\end{array}$} & \multirow[t]{3}{*}{ (14) } \\
\hline & & Partially & 0.67 & & \\
\hline & & Uncompleted & 1 & & \\
\hline \multirow[t]{3}{*}{13} & \multirow{3}{*}{$\begin{array}{l}\text { Children under } 12 \\
\text { months received } 3 \text { rd } \\
\text { pentavalent vaccine }\end{array}$} & Completed & 0.33 & \multirow{3}{*}{$\begin{array}{l}\text { Households with high } \\
\text { deficit rate of } \\
\text { immunization are very } \\
\text { highly vulnerable to risks } \\
\text { of epidemics }\end{array}$} & \multirow[t]{3}{*}{ (14) } \\
\hline & & Partially & 0.67 & & \\
\hline & & Uncompleted & 1 & & \\
\hline \multirow[t]{2}{*}{14} & \multirow{2}{*}{$\begin{array}{l}\text { Mosquito bed net } \\
\text { utilized by the } \\
\text { recommended groups }\end{array}$} & Yes & 0.5 & \multirow{2}{*}{$\begin{array}{l}\text { Under } 5 \text { years children and } \\
\text { pregnant women }\end{array}$} & \multirow[t]{2}{*}{ (36) } \\
\hline & & No & 1.00 & & \\
\hline \multirow[t]{2}{*}{15} & \multirow{2}{*}{$\begin{array}{l}\text { Source of drinking } \\
\text { water }\end{array}$} & $\begin{array}{l}\text { Improved } \\
\text { water source }\end{array}$ & 0.5 & \multirow{2}{*}{$\begin{array}{l}\text { Unimproved water sources } \\
\text { are considered producing } \\
\text { greater vulnerability }\end{array}$} & \multirow[t]{2}{*}{ (36) } \\
\hline & & $\begin{array}{l}\text { Unimproved } \\
\text { water source }\end{array}$ & 1 & & \\
\hline \multirow[t]{3}{*}{16} & \multirow{3}{*}{$\begin{array}{l}\text { Water coverage per } \\
\text { capita per day. }\end{array}$} & $<15$ liters & 1 & \multirow{3}{*}{$\begin{array}{l}\text { A household with low } \\
\text { water coverage is very } \\
\text { highly vulnerable water- } \\
\text { related diseases }\end{array}$} & \multirow[t]{3}{*}{ (5) } \\
\hline & & 15-20 liters & 0.66 & & \\
\hline & & $>20$ liters & 0.33 & & \\
\hline \multirow[t]{3}{*}{17} & \multirow[t]{3}{*}{ Water safety } & No treatment & 1 & \multirow{3}{*}{$\begin{array}{l}\text { Standard water treatment } \\
\text { measures are the } \\
\text { chlorination and boiling }\end{array}$} & \multirow[t]{3}{*}{ (5) } \\
\hline & & Improper & 0.66 & & \\
\hline & & Proper & 0.33 & & \\
\hline
\end{tabular}




\begin{tabular}{|c|c|c|c|c|c|}
\hline $\begin{array}{l}\text { S. } \\
\text { No }\end{array}$ & Indicators & classes & $\begin{array}{l}\text { Weighing } \\
\text { scale }\end{array}$ & Interpretation & $\begin{array}{l}\text { Selected } \\
\text { indicators } \\
\text { used by } \\
\text { other } \\
\text { studies }\end{array}$ \\
\hline \multirow[t]{5}{*}{18} & \multirow[t]{5}{*}{ Type of latrine } & \multirow{2}{*}{$\begin{array}{l}\text { Ventilation } \\
\text { improved } \\
\text { latrine }\end{array}$} & 0.33 & \multirow{5}{*}{$\begin{array}{l}\text { Unimproved toilet facilities } \\
\text { are more vulnerable than } \\
\text { improved facilities, as } \\
\text { microbiological } \\
\text { contamination }\end{array}$} & \multirow[t]{5}{*}{$(5)$} \\
\hline & & & 0.66 & & \\
\hline & & $\begin{array}{l}\text { Pit latrine } \\
\text { with slab }\end{array}$ & 1 & & \\
\hline & & $\begin{array}{l}\text { Pit latrine } \\
\text { without a } \\
\text { slab }\end{array}$ & & & \\
\hline & & Others & & & \\
\hline \multirow[t]{3}{*}{19} & \multirow{3}{*}{$\begin{array}{l}\text { Safe disposal of } \\
\text { children feces }\end{array}$} & In toilet & 0.33 & \multirow{3}{*}{$\begin{array}{l}\text { household disposal the } \\
\text { children feces on the floor } \\
\text { are more vulnerable to the } \\
\text { risk of diseases }\end{array}$} & \multirow[t]{3}{*}{$(45)$} \\
\hline & & In a potty & 0.66 & & \\
\hline & & On the floor & 1 & & \\
\hline \multirow[t]{2}{*}{20} & \multirow{2}{*}{$\begin{array}{l}\text { Household received } \\
\text { washing soap in last } \\
\text { month }\end{array}$} & Yes & 0.5 & \multirow{2}{*}{$\begin{array}{l}\text { Household not received } \\
\text { soap in the regular base } \\
\text { are more vulnerable to } \\
\text { health risk }\end{array}$} & \multirow[t]{2}{*}{$(45)$} \\
\hline & & No & 1 & & \\
\hline \multirow[t]{2}{*}{21} & \multirow{2}{*}{$\begin{array}{l}\text { Household received } \\
\text { hygiene kits in last } \\
\text { month }\end{array}$} & Yes & 0.5 & \multirow{2}{*}{$\begin{array}{l}\text { Household did not receive } \\
\text { hygiene kits in a camp } \\
\text { setting become more } \\
\text { vulnerable to risk }\end{array}$} & \multirow[t]{2}{*}{$(46)$} \\
\hline & & No & 1 & & \\
\hline \multirow[t]{2}{*}{22} & \multirow{2}{*}{$\begin{array}{l}\text { Availability of the } \\
\text { solid waste } \\
\text { management system } \\
\text { in camp }\end{array}$} & Yes & 0.5 & \multirow{2}{*}{$\begin{array}{l}\text { The absence of solid waste } \\
\text { management increases the } \\
\text { vulnerability risk of } \\
\text { diseases }\end{array}$} & \multirow[t]{2}{*}{$(46)$} \\
\hline & & No & 1 & & \\
\hline \multicolumn{6}{|c|}{ 3. public health Capacity indicators (phC) } \\
\hline \multirow[t]{2}{*}{1} & \multirow{2}{*}{$\begin{array}{l}\text { A household with } \\
\text { other } \\
\text { sources/multiple } \\
\text { sources of income of } \\
\text { income }\end{array}$} & Yes & 1 & \multirow{2}{*}{$\begin{array}{l}\text { Multiple sources of income } \\
\text { will increase in case if one } \\
\text { of the sources is cut of the } \\
\text { household can survive on } \\
\text { another }\end{array}$} & \multirow[t]{2}{*}{ (9) } \\
\hline & & No & 0 & & \\
\hline \multirow[t]{2}{*}{2} & \multirow{2}{*}{$\begin{array}{l}\text { A household with } \\
\text { family member } \\
\text { employed outside } \\
\text { this camp or state }\end{array}$} & Yes & 1 & \multirow{2}{*}{$\begin{array}{l}\text { Households with a family } \\
\text { member employed outside } \\
\text { the camp will be high } \\
\text { capacity. }\end{array}$} & \multirow[t]{2}{*}{$(5)$} \\
\hline & & No & 0 & & \\
\hline \multirow[t]{2}{*}{3} & \multirow{2}{*}{$\begin{array}{l}\text { Household having } \\
\text { any kind of savings }\end{array}$} & Yes & 1 & \multirow{2}{*}{$\begin{array}{l}\text { Saving will increase the } \\
\text { capacity of the households } \\
\text { to cope with emergency } \\
\text { and will help in recovery }\end{array}$} & \multirow[t]{2}{*}{ (9) } \\
\hline & & No & 0 & & \\
\hline
\end{tabular}




\begin{tabular}{|c|c|c|c|c|c|}
\hline $\begin{array}{l}\text { S. } \\
\text { No }\end{array}$ & Indicators & classes & $\begin{array}{l}\text { Weighing } \\
\text { scale }\end{array}$ & Interpretation & $\begin{array}{l}\text { Selected } \\
\text { indicators } \\
\text { used by } \\
\text { other } \\
\text { studies }\end{array}$ \\
\hline 4 & $\begin{array}{l}\text { Household } \\
\text { awareness about } \\
\text { safe water- }\end{array}$ & $\begin{array}{l}\text { High } \\
\text { knowledge } \\
\text { Medium } \\
\text { knowledge } \\
\text { Low } \\
\text { knowledge } \\
\text { No } \\
\text { knowledge }\end{array}$ & $\begin{array}{l}1 \\
0.67 \\
0.33 \\
0\end{array}$ & $\begin{array}{l}\text { Awareness about safe } \\
\text { water will increase } \\
\text { household capacity to } \\
\text { cope with diarrheal } \\
\text { diseases during emergency }\end{array}$ & (47) \\
\hline 5 & $\begin{array}{l}\text { Availability of health } \\
\text { services and easy } \\
\text { accessed by } \\
\text { household }\end{array}$ & $\begin{array}{l}\text { Available } \\
\text { Not } \\
\text { Available }\end{array}$ & $\begin{array}{l}1 \\
0\end{array}$ & $\begin{array}{l}\text { Availability and } \\
\text { accessibility to health } \\
\text { services will increase } \\
\text { household capacity } \\
\text { against emergency } \\
\text { diseases }\end{array}$ & (36) \\
\hline 6 & $\begin{array}{l}\text { Household } \\
\text { awareness about the } \\
\text { way of diarrheal } \\
\text { transmitted. }\end{array}$ & $\begin{array}{l}\text { High } \\
\text { knowledge } \\
\text { Medium } \\
\text { knowledge } \\
\text { Low } \\
\text { knowledge } \\
\text { No } \\
\text { knowledge }\end{array}$ & $\begin{array}{l}1 \\
0.67 \\
0.33 \\
0\end{array}$ & $\begin{array}{l}\text { Awareness about diarrhea } \\
\text { transmitted will help to } \\
\text { prepare for diarrhea control }\end{array}$ & (46) \\
\hline 7 & $\begin{array}{l}\text { Household } \\
\text { awareness about the } \\
\text { way of diarrheal } \\
\text { prevention }\end{array}$ & $\begin{array}{l}\text { High } \\
\text { knowledge } \\
\text { Medium } \\
\text { knowledge } \\
\text { Low } \\
\text { knowledge } \\
\text { No } \\
\text { knowledge }\end{array}$ & $\begin{array}{l}1 \\
0.67 \\
0.33 \\
0\end{array}$ & $\begin{array}{l}\text { Awareness about diarrhea } \\
\text { prevention can increase the } \\
\text { coping capacity of the } \\
\text { household against diarrhea } \\
\text { during an emergency. }\end{array}$ & (46) \\
\hline 8 & $\begin{array}{l}\text { Household having } \\
\text { any kind of bed net }\end{array}$ & $\begin{array}{l}\text { Yes } \\
\text { No }\end{array}$ & $\begin{array}{l}1 \\
0\end{array}$ & $\begin{array}{l}\text { Bed net will help } \\
\text { households to better cope } \\
\text { with diseases that } \\
\text { transmitted through } \\
\text { vectors }\end{array}$ & (36) \\
\hline
\end{tabular}




\begin{tabular}{|c|c|c|c|c|c|}
\hline $\begin{array}{l}\text { S. } \\
\text { No }\end{array}$ & Indicators & classes & $\begin{array}{l}\text { Weighing } \\
\text { scale }\end{array}$ & Interpretation & $\begin{array}{l}\text { Selected } \\
\text { indicators } \\
\text { used by } \\
\text { other } \\
\text { studies }\end{array}$ \\
\hline \multirow[t]{2}{*}{9} & \multirow{2}{*}{$\begin{array}{l}\text { The household has } \\
\text { taken personal } \\
\text { measures to be } \\
\text { prepared for diarrhea } \\
\text { and malaria control }\end{array}$} & Yes & 1 & \multirow{2}{*}{$\begin{array}{l}\text { Households taken personal } \\
\text { measures against diarrhea } \\
\text { will be high capacity and } \\
\text { avoid the impacts or } \\
\text { consequences of diarrhea } \\
\text { and malaria }\end{array}$} & \multirow[t]{2}{*}{$(36)$} \\
\hline & & No & 0 & & \\
\hline \multirow[t]{2}{*}{10} & \multirow{2}{*}{$\begin{array}{l}\text { Household has been } \\
\text { involved in planning } \\
\text { for disease } \\
\text { prevention and } \\
\text { management }\end{array}$} & Yes & 1 & \multirow{2}{*}{$\begin{array}{l}\text { Household involved the } \\
\text { emergency planning will be } \\
\text { highly capacity }\end{array}$} & \multirow[t]{2}{*}{ (19) } \\
\hline & & No & 0 & & \\
\hline
\end{tabular}

\section{Data analysis}

Composite index methodologies have evolved to meet a wide range of purposes and to inform the authorities to take particular interventions or specific decisions. (25). Also, the indices can be very useful tools for analysis because of their ability to summarize more complicated technical data into a simpler way (35). In this regard, a composite index method was used in this study to develop a public health risk index by combining the three main component indexes e.g., public health hazard index (phHI), public health vulnerability index (phVI), and public heath capacity index (phCl). After proper weights assigned to classes of phenomenon for each indicator, the index for each component was calculated using Eq. 2 . In this case, the original data sets were transformed to respective weights for computation of the composite index of each component as used by some researchers (36).

$$
C I=\left(W_{1}+W_{2}+W_{3} \ldots \ldots+W_{n}\right) / n=
$$

$$
\sum_{i=1}^{n} W_{i} / n
$$

Where $\mathrm{Cl}$ is the composite index, $W_{1}$ to $W_{n}$ are respective transformed values assigned to Following this general principle, the public health Hazard Index (phHI), public health Vulnerability Index (phVI), public health Capacity Index (phCl), were calculated. 
Public Health Hazard Index (phHI) $=\sum_{i=1}^{2} H W_{i} / n \quad(\mathrm{n}=2)$

Public Health Vulnerability Index $(\mathrm{phVI})=\sum_{i=1}^{22} V W_{i} / n \quad(\mathrm{n}=22)$

Public Health Capacity Index $(\mathrm{phCI})=\sum_{i=1}^{10} C W_{i} / n \quad(\mathrm{n}=10)$

\section{Public Health Risk Index (PHRI) Model}

A three-phase methodology approach was used to construct the final public health risk index model. Firstly, indicators for hazard, vulnerability, and capacity were identified and primary data was collected from households through the questionnaire. The second phase focuses on weight located/assigned to each indicator. Thirdly, the Public Health Risk index (PHRI) for each household in the study area was calculated using Eq. 3. In this case, the original values from the public health hazard index (phHI), public health vulnerability index $(\mathrm{phVl})$, and public health capacity index $(\mathrm{phCl})$ were multiplied to generate the final public health risk index through the following equation.

Public Health Risk Index (PHRI) $=\frac{\text { Public Health Hazard Index (phHI) } X \text { Public Helath Vulnerability Index (phVI) }}{\text { Public Health Capacity Index (phCI) }} \quad$ Eq.3

After the creation of the public health risk index model at the IDPs camp level the overall mean and standard deviation techniques methods were used to group the households in four levels such as low, moderate, high and very high, so as to understanding the degree of public health risk at household level for comparative analysis with respect to public health risks. For comparative analysis, ANOVA (analysis of variance) test was used to determine the difference among the camp.

\section{Results And Discussion}

The development of the public health risk index was based on the composite index model mentioned in the methodology. Twenty-two indicators were used for vulnerability, ten indicators were used for capacity assessment and two indicators were used for health hazards. This methodology provides clarity to indicators used under different components, such as hazard, vulnerability, and capacity. The concepts of disaster risk were applied to develop public health risk index through disaster components these include risk, hazard, vulnerability, and capacity assessment.

The analysis shows that the highest level of illiteracy rate among the IDPs was reported by Shangil Tobaya household respondents at $48.5 \%$ because this camp is located in very remote area and those living in this camp originally from rural areas and did not get education opportunities even before the conflict. While the lowest illiteracy rate was reported in Abu Shock camp at $42.6 \%$. Approximately $60 \%$ of 
the respondents earning less than 1,250 Sudanese pounds (equals 40 United States dollars) a month which indicates that the highest poverty rate among the IDPs.

\section{Level Of Hazard}

The study revealed that health hazard was most severe in Shangil Tobaya camp were around $21.2 \%$ of households were very high exposure to public health hazards, followed by Zamzam $13.8 \%$ and Abu Shock camp was $1.5 \%$ as showed in (Fig. 4$)$. The Significant difference $\left(X^{2}=29.145\right.$, $p$-value 0.000$)$ was observed among households regarding the frequency and severity of public health hazards. Moreover, the analysis indicated that the average of the public hazard index value was found to be $0.7,0.66$ and 0.55 at household level in Shangil Tobaya, Zamzam and Abu Shouk, respectively. Many factors made households of Shangil Tobaya as the most exposed to the health hazard. First, IDPs in Shangil Tobaya camp are living in a small camp characterized by overcrowding, poor living conditions, Inadequate basic services such as health, water, sanitation, and food. These findings were supported by the secondary data set, which was collected from the State Ministry of Health North Darfur and revealed that the Shangil Tobaya camp experienced seven infectious disease outbreaks from 2006 to 2017, followed by Zamzam six outbreaks and Abu Shouk camp five infectious disease outbreaks (32).Second, the health hazard in Zamzam also near to high because this camp accommodates a high number of the IDPs; it is the largest camp in the Darfur region and still only open camp. Health hazards only convert into epidemic diseases due to low-level capacity as preparedness and mitigation measures to cope with health hazard (12). Despite, $13.8 \%$ of Zamzam households were very high exposure to the health hazard, but due to the better health services in Zamzam camp, in addition to quick response usually made by INGOs and government that prevent the spread of several outbreaks and automatically reduce the severity of the disease's outbreaks. The health hazard in Abu Shouk households was found to be low because this camp was established close to an urban area that helps IDPs to have access to primary and secondary health services. Furthermore, the environmental health condition inside the Abu Shouk camp is better than Zamzam and Shangil Tobaya

\section{Level Of Vulnerability:}

The study shows that the households of Shangil Tobaya camp were the most vulnerable $(69.7 \%)$ of the household's range between high to very high followed by Zamzam camp (52.5\%) and Abu Shock (42.7\%.) as showed in (Table 2). The factors that increased the vulnerability of IDPs in Shangil Tobaya include lack inadequate of the health services, lack of access to safe water and proper sanitation and hygiene, lack of awareness and high illiteracy rate. The average public health vulnerability value was found to be $0.75,0.73$ and 0.72 at household level in Shangil Tobaya, Zamzam and Abu Shouk camp, respectively. Households were categorized into four public health vulnerability levels according to transformed composite indices values used the overall mean and standard deviation (SD) techniques as shown in (Table 2). One -way analysis of variance (ANOVA) test was confirmed that a significant difference ( $F=$ 4.048, $p$-value $=0.018$ ) existed in households in three IDP. To further specify the exact difference between camps, the multiple comparisons between the camps (pairs) were done using Least Significant 
Difference (LSD), and reflected there was a highly significant difference between Abu Shouk and Shangil Tobaya (P-value $=0.006)$, whereas no significant difference was detected between Zamzam and Shangil Tobaya (P-value $=0.086)$; and between Abu Shock and Zamzam $(P$-value $=0.054)$. In this regard, a Chisquare test was conducted to understand why difference does exist in the two camps as it was pointed out by LSD. However, the chi-square test revealed that eight out of 22 indicators appeared to have made a significant difference between the Shangil Tobaya and Abu Shock camps. These include educational level, walking time to health facilities, frequency of visit by pregnant women to antenatal care services, place of delivery, women delivering their children with help of skilled birth attendants, sources of water, latrine type and safe disposal of child feces. Figure $5,6 \& 7$ ) shows the current status of types of latrines in camp, health facility and water resources in camps.

Table 2

Household health vulnerability level at three IDP camps

\begin{tabular}{|c|c|c|c|c|c|c|c|c|}
\hline \multirow[t]{3}{*}{ Level } & \multirow{2}{*}{\multicolumn{2}{|c|}{ Abu Shouk }} & \multirow{2}{*}{\multicolumn{2}{|c|}{ Zamzam }} & \multicolumn{4}{|c|}{ Shangil } \\
\hline & & & & & \multicolumn{2}{|c|}{ Tobaya } & \multicolumn{2}{|c|}{ ANOV test } \\
\hline & Freq & $\%$ & Freq & $\%$ & Freq & $\%$ & F-Value & P-Value \\
\hline Low & 19 & 27.9 & 41 & 18.2 & 2 & 6.1 & 4.048 & 0.018 \\
\hline Moderate & 20 & 29.4 & 66 & 29.3 & 8 & 24.2 & & \\
\hline High & 22 & 32.4 & 70 & 31.1 & 15 & 45.5 & & \\
\hline Very high & 10 & 10.3 & 48 & 21.4 & 8 & 24.2 & & \\
\hline Total & 68 & 100 & 225 & 100 & 33 & 100 & & \\
\hline
\end{tabular}

Low $<0.68$, Moderate $(0.68$ to $<0.73)$, High $(0.74$ to $<0.79)$, Very high $<0.79$

\section{Level Of Capacity}

The capacity analysis is one of the important pillars of risk assessment. The average capacity value was 0.45 in Zamzam, 0.41 in Shangil Tobaya in and 0.40 in Abu Shouk This study indicated that no significant difference $\left(X^{2}=5.059\right.$, P-value 0.539$)$ in the level of capacity was observed between the three IDPs camps as shown in (Table 3). The reasons behind the near to the same level of capacity at households level because all IDPs are highly impacted by the conflict and lost everything in terms of their works and assets these assets include social, financial, human, natural and physical assets that made them very weak in terms of capacity. Overall, about $34.2 \%$ of the households had dependents on only one source of income and only $13.2 \%$ of households had a member working outside the camp. Almost, $94.8 \%$ of households did not have any kind of saving. About $50.9 \%$ of the households had a lack or no knowledge regard the safe water which that let them the low capacity to prevent the epidemic of communicable diseases in humanitarian settings. $70.9 \%$ of the households had faced a lack of access to basic health services. $66.9 \%$ of households had low or no knowledge regard the way of diarrhea transmission and only $12 \%$ of households had high knowledge about diarrhea prevention. Only $34 \%$ of 
households are taken personal measures to be prepared for diarrhea and malaria control. About $86.6 \%$ of households did not involve by humanitarian partners in planning for disease prevention and management that why let them the weak capacity to deal with epidemic disease. All these results indicated that capacity levels for all targeted camps were very weak that let them every year have been affected by outbreak of communicable diseases.

Table 3

Household capacity level at the targeted camps

\begin{tabular}{|c|c|c|c|c|c|c|c|c|}
\hline \multirow[t]{2}{*}{ Level } & \multicolumn{2}{|c|}{ Abu Shock } & \multicolumn{2}{|c|}{ Zamzam } & \multicolumn{2}{|c|}{ Shangil Tobaya } & \multirow{2}{*}{$\begin{array}{l}\text { Chi-Square } \\
5.059\end{array}$} & \multirow{2}{*}{$\begin{array}{l}\text { P- Value } \\
0.539\end{array}$} \\
\hline & Freq & $\%$ & Freq & $\%$ & Freq & $\%$ & & \\
\hline Low & 10 & 14.7 & 35 & 15.6 & 2 & 6.1 & & \\
\hline Moderate & 21 & 30.9 & 80 & 35.6 & 13 & 39.3 & & \\
\hline High & 24 & 35.3 & 74 & 32.8 & 15 & 45.5 & & \\
\hline Very high & 13 & 19.1 & 36 & 16.0 & 3 & 9.1 & & \\
\hline Total & 68 & 100 & 225 & 100 & 33 & 100 & & \\
\hline
\end{tabular}

The degree of public health risk at households' level in three IDPs camps was calculated using the equation mentioned in the previous section (Eq. 3). The study reflected that households of Shangil Tobaya camp were the most at high risk to epidemic of communicable diseases, about (24.2\%) of Shangil Tobaya households at very high risk followed by Zamzam (15.6\%) and Abu Shock camp (10.3\%) as shown in (Table 4). The reasons that led Shangil Tobaya become at high risk because of their high vulnerability factors such as low level of income, lack of access to basic services, low level of education and their location in rural area that prevent the IDPs to get the work opportunities to improve their capacity and decrease their vulnerability. The average risk value was 0.71 in Abu Shock, 0.99 in Zamzam and 1.00 in Shangil Tobaya. In addition, the study reflected that there is a highly significant difference $\left(\mathrm{X}^{2}\right.$ $=18.133$, P-value 0.006 ) was observed among households in three camps to public health risk as shown in (Table 4). Moreover, the analysis showing that the smallest camp in rural area is a very high risk of epidemic diseases, followed by the biggest camp then middle camp. IDPs in Abu Shock (around the urban area) had the lowest public health risks compared to the other two camps in rural areas, due to their high level of awareness regarding disease transmission, prevention and control, easy access to the services, and their better income. Our finding is analogous with the study conducted in war-affected area in Afghanistan; the result shows that the IDPs living around urban areas having better incomes and easy access to basic services than those living in rural areas (37). The hazard and vulnerability were very high for all affected populations in targeted camps. Vulnerability in three IDP communities is varying. Shangil is Tobaya the most vulnerable, followed by Zamzam and Abu Shock. This finding was supported by the secondary data set, which was taken from the WHO and Ministry of Health annual report revealed that 
the high prevalence of diarrhea diseases recorded in Shangil Tobaya camp compared to Abu Shock and Zamzam (Fig. 8).

Table 4

Households public health risk level at three targeted camps

\begin{tabular}{|c|c|c|c|c|c|c|c|c|}
\hline \multirow[t]{2}{*}{ Level } & \multicolumn{2}{|c|}{ Abu Shock } & \multicolumn{2}{|c|}{ Zamzam } & \multicolumn{2}{|c|}{ Shangil Tobaya } & \multirow{2}{*}{$\begin{array}{c}\text { Chi-Square } \\
18.133\end{array}$} & \multirow{2}{*}{$\begin{array}{l}\text { P-Value } \\
0.006\end{array}$} \\
\hline & Freq & $\%$ & Freq & $\%$ & Freq & $\%$ & & \\
\hline Low & 19 & 27.9 & 34 & 15.1 & 2 & 6.1 & & \\
\hline Moderate & 28 & 41.2 & 68 & 30.2 & 13 & 39.4 & & \\
\hline High & 14 & 20.6 & 88 & 39.1 & 10 & 30.3 & & \\
\hline Very high & 7 & 10.3 & 35 & 15.6 & 8 & 24.2 & & \\
\hline Total & 68 & 100 & 225 & 100 & 33 & 100 & & \\
\hline
\end{tabular}

Average values of the components of the public health risk reflected a difference among the three IDPS camps to shows we used, Radar chart to provides an easy way of understanding public health risk and its components (Fig. 9). Health hazard was most severe in Shangil Tobaya camp, followed by Zamzam, and Abu Shock camp. The vulnerability was found to be high in Shangil Tobaya followed by Zamzam and Abu Shock camp. Capacity was found to be near to the same in all households at three IDP camps. The risk was found to be very high within Shangil Tobaya households followed by Zamzam, and Abu Shock. The evidence from the secondary report reflected that Shangil Tobaya Camp was experiencing several diseases outbreaks in the previous years $(2014,2015,2016)$ in addition to the recent one is acute watery diarrhea (Cholera) affected the camp in 2017, that resulted in 213 cases and 14 deaths, as shown in (Fig. 10).

\section{Conclusions}

This paper describes the development of a public health risk assessment model (PH-RAM) that aims to assess the public health risk of internally displaced person (IDPs) in three camps in North Darfur of Sudan and to enhance public health risk assessment methods and tools at the national and local level, and suggest appropriate measures to mitigate and reduce the health risk and vulnerability of the IDPs. The suggested model was developed through incorporated and combining the three main component indexes (public health hazard, vulnerability, and capacity index). This paper highlights very important interventions that can be implemented by the government of Sudan and international organizations to reduce the vulnerability and health risks of the IDP. The method described in this paper is flexible, actionable and can be replicated for other emergency areas in the context of IDPs at all mentioned levels.

The finding of this study indicated that households of Shangil Tobaya camp were the most at high risk to epidemic of communicable diseases $(24.2 \%)$ of households in this camp at very health risk followed by Zamzam (15.6\%) and Abu Shouk camp (10.3\%). Also, the analysis shows that the main factors that 
increased the vulnerability of the IDPs to health risks are lack of access to health services, unsafe water, poor sanitation, low income, and low level of education. This finding is consistent with a study conducted in Pakistan to assess public health status of IDPs and Refugees, which confirming that the lack of access to water, sanitation, and healthcare are the main reasons of high prevalence of diseases among the IDPs and refugees from Afghanistan $[14 ; 21]$.

The study recommended that mitigation and prevention measures could be implemented to reduce the factors that are shown increased the vulnerability of the IDPs to health risks. The result of this study could be helping the government of Sudan to develop appropriate public health risk reduction strategies in the context of IDPs. Also, the comprehensive studied indicators could be used by other researchers in the field of public health risk. Lastly, the suggested model could be served as public health risk assessment tools in the context of IDPs or refugees at the local, national, and regional levels.

\section{List Of Abbreviations}

PH-RAM: Public Health Risk Assessment Model; IDPs: Internally Displaced Persons; UNISDR: United Nations Office for Disaster Risk Reduction; WHO: World Health Organization; phR: public health Risk; phH: public health Hazard; phV: public health Vulnerability; phC: public health Capacity; Cl: Composite Index; PHRI: Public Health Risk Index.

\section{Declarations}

\section{Authors' contributions}

ME and PI designed the study, ME analyzed the data and drafted the manuscript, AH revise the manuscript and added valuable comments during the revise. All authors read and approved the final manuscript.

\section{Authors information's}

${ }^{1}$ Department of Disaster Preparedness Mitigation and Management, Asian Institute of Technology, Bangkok, Thailand. ${ }^{2}$ Department of Disaster Preparedness, Mitigation and Management. Asian Institute of Technology, Bangkok, Thailand. ${ }^{3}$ World Health Organization (WHO) Regional Office for the Eastern Mediterranean, Eastern Mediterranean Public Health Network, Jordan.

\section{Acknowledgements}

Not applicable

\section{Competing interests}

The authors declare that they have no competing interests. 


\section{Availability of data}

Data are available from the corresponding author upon reasonable request

\section{Consent for publication}

Not applicable.

\section{Ethics approval and consent to participate}

Not applicable.

\section{Funding}

ME acknowledges that the Asian Institute of Technology, Thailand; and Public Health Institute, Sudan was supporting this study through research funds, and granting PhD. Scholarship to the first author.

\section{References}

1. Owoaje E, Uchendu O, Ajayi T, Cadmus E. A review of the health problems of the internally displaced persons in Africa. Niger Postgrad Med J. 2016;23(4):161.

2. IDMC. Global report on internal displacement [Internet]. 2018. Available from: https://reliefweb.int/report/world/2018-global-report-internal-displacement-grid-2018, (accessed 9 April 2019).

3. UNOCHA. Sudan humanitarian needs overview report [Internet]. 2018. Available from: https://reliefweb.int/.../sudan/sudan-2018-humanitarian-needs-overview, (accessed 19 Feburay 2020).

4. Degomme O, Guha-Sapir D. Patterns of mortality rates in Darfur conflict. Lancet. 2010;375(9711):294-300.

5. Mahmoud EM, Pal I, Ahmad MM. Assessing public health risk factors for internally displaced households in North Darfur, Sudan. 2019;

6. Chan EYY, Huang Z, Lam HCY, Wong CKP, Zou Q. Health vulnerability index for disaster risk reduction: Application in belt and road initiative (BRI) region. Int J Environ Res Public Health. 2019;16(3).

7. Thomas SL, Thomas SDM. Displacement and health. Br Med Bull. 2004;69:115-27.

8. Waring SC, Brown BJ. The threat of communicable diseases following natural disasters: A public health response. Disaster Manag Response. 2005;3(2):41-7.

9. Rana IA, Routray JK. Integrated methodology for flood risk assessment and application in urban communities of Pakistan. Nat Hazards. 2018;91(1):239-66.

10. Abrahams J, Smallwood C, Anacia A, Wannous C, Espinosa L, Ciotti M, et al. Health Aspect in Disaster Risk Assessment [Internet]. 2017. Available from:

https://www.preventionweb.net/files/52828_ehealthaspect[1].pdf. 
11. UNISDR. Terminology on Disaster Risk Reduction [Internet]. International Stratergy for Disaster Reduction (ISDR). 2009. Available from: https://www.unisdr.org/we/inform/terminology\%0A, (accessed 5 March 2019).

12. UNDP. Mainstreaming Disaster Risk Reduction into Health: Strategies, Methodologies and Tools [Internet]. Vol. 002. 2012. Available from:

https://smartnet.niua.org/sites/default/files/resources/DRR-health.pdf. (accessed 23 January 2020).

13. Smith KF, Goldberg M, Rosenthal S, Carlson L, Chen J, Chen C, et al. Global rise in human infectious disease outbreaks. J R Soc Interface. 2014;11(101):1-6.

14. Ahmad J, Morshed Ahmad M, Sadia H, Ahmad A. Using selected global health indicators to assess public health status of population displaced by natural and man-made disasters. Int J Disaster Risk Reduct. 2017;22:228-37.

15. Füssel HM. Vulnerability: A generally applicable conceptual framework for climate change research. Glob Environ Chang. 2007;17(2):155-67.

16. Sadia H, Iqbal MJ, Ahmad J, Ali A, Ahmad A. Gender-sensitive public health risks and vulnerabilities' assessment with reference to floods in Pakistan. Int J Disaster Risk Reduct [Internet]. 2016;19:47-56. Available from: http://dx.doi.org/10.1016/j.ijdrr.2016.08.024

17. Michael J, Toole M. The Rapid Assessment of Health Problems in Refugee and Displaced Populations. Med Glob Surviv [Internet]. 1994;1(4):200-6. Available from: http://ippnw.org/pdf/mgs/1-4-toole.pdf, (accessed 11 January 2020).

18. Roberts B, Odong VN, Browne J, Ocaka KF, Geissler W, Sondorp E. An exploration of social determinants of health amongst internally displaced persons in northern Uganda. Confl Health. 2009;3(1):1-11.

19. Schnitter R, Verret M, Berry P, Fook TCT, Hales S, Lal A, et al. An assessment of climate change and health vulnerability and adaptation in dominica. Int J Environ Res Public Health. 2019;16(1).

20. WHO. Climate Change and Human Health - Risks and Responses [Internet]. 2000. Available from: http://www.who.int/globalchange/environment/en/ccSCREEN.pdf?ua=1, (accessed 11 April 2020).

21. Mohanty A, Hussain M, Mishra M, Kattel DB, Pal I. Exploring community resilience and early warning solution for flash floods, debris flow and landslides in conflict prone villages of Badakhshan, Afghanistan. Int J Disaster Risk Reduct [Internet]. 2019;33(July 2018):5-15. Available from: https://doi.org/10.1016/j.ijdrr.2018.07.012

22. Pal I, Bhatia S. Disaster risk governance and city resilience in Asia-Pacific region [Internet]. Science and Technology in Disaster Risk Reduction in Asia: Potentials and Challenges. Elsevier Inc.; 2017. 137-159 p. Available from: http://dx.doi.org/10.1016/B978-0-12-812711-7.00009-2

23. Fadlallah MA, Pal I, Hoe VC. Determinants of perceived risk among artisanal gold miners: A case study of Berber locality, Sudan. Extr Ind Soc [Internet]. 2020;(September 2019):0-1. Available from: https://doi.org/10.1016/j.exis.2020.03.006 
24. Thanvisitthpon N, Shrestha S, Pal I, Ninsawat S, Chaowiwat W. Assessment of flood adaptive capacity of urban areas in Thailand. Environ Impact Assess Rev [Internet]. 2020;81(December 2019):106363. Available from: https://doi.org/10.1016/j.eiar.2019.106363

25. Baptista SR. Design and use of composite indices in assessment of climate change vulnerability and resilience. United States Agency Int Dev. 2014;(July):53pp.

26. Fuchs S, Birkmann J, Glade T. Vulnerability assessment in natural hazard and risk analysis: Current approaches and future challenges. Nat Hazards. 2012;64(3):1969-75.

27. Pal I, Tularug P, Jana SK, Pal DK. Risk Assessment and Reduction Measures in Landslide and Flash Flood-Prone Areas: A Case of Southern Thailand (Nakhon Si Thammarat Province). Integrating disaster science and management: Global case studies in mitigation and recovery. [Internet]. Integrating Disaster Science and Management. Elsevier Inc.; 2018. 295-308 p. Available from: http://dx.doi.org/10.1016/B978-0-12-812056-9/00017-8

28. Pramanik M, Diwakar AK, Dash P, Szabo S, Pal I. Conservation planning of cash crops species (Garcinia gummi-gutta) under current and future climate in the Western Ghats, India. Environ Dev Sustain [Internet]. 2020;(0123456789). Available from: https://doi.org/10.1007/s10668-020-00819-6

29. WHO. International Health Regulation [Internet]. 2005. Available from: https://www.who.int/ihr/9789241596664/en/, (accessed 24 January 2020).

30. Hammer CC, Brainard J, Hunter PR. Risk factors and risk factor cascades for communicable disease outbreaks in complex humanitarian emergencies: A qualitative systematic review. BMJ Glob Heal. 2018;3(4):1-10.

31. Central Bureau of Statistic (CBS). Sudan multiple indicator cluster survey,2014 [Internet]. Khartoum; 2014. Available from: microdata.worldbank.org > ... > MICS > Multiple Indicator Cluster Survey 2014, (accessed 20 March 2019).

32. State Ministry of Health. Internally displaced persons (IDPs) camps profile. 2017. unpublised data collected on (20 March 2018).

33. Yamane T. Statistics: An Introductory Analysis. New York: Harper and Row; 1967.

34. Umulisa C. Sampling Methods and Sample Size Calculation for the SMART Methodology June 2012 Table of Contents. In: University [Internet]. 2012. p. 20-30. Available from: https://www.humanitarianresponse.info/sites/www.../Sampling_Paper_June_2012.pdf, (accessed 5 January 2018).

35. Sullivan C, Meigh J. Targeting attention on local vulnerabilities using an integrated index approach: The example of the climate vulnerability index. Water Science and Technology, 51(5),. Water Sci Technol. 2005;51(5):69-78.

36. Abbas HB, Routray JK. Vulnerability to flood-induced public health risks in Sudan. Disaster Prev Manag. 2014;23(4):395-419.

37. UNHCR/World Bank. Research Study on IDPs in urban settings - Afghanistan [Internet]. 2011. Available from: http://documents.worldbank.org/curated/en/2011/05/16257782/afghanistanresearch-study-idps-urban-settings (accessed 5 January 2020). 
38. Peters R, Kricun H, Hipper T, Chernak E. The Pennsylvania Public Health Risk Assessment Tool Instructions for Using the Pennsylvania Public Health Risk Assessment Tool Workbook [Internet]. 2013. Available from: https://drexel.edu/dornsife/research/centers-programs-projects/center-forpublic-health-readiness-communication/our-projects/phrat/

39. Srikuta P, Inmuong U, Inmuong Y, Bradshaw P. Health Vulnerability of Households in Flooded Communities and Their Adaptation Measures. Asia-Pacific J Public Heal. 2015;27(7):743-55.

40. Jha R, Kang W, Nagarajan HK, Pradhan KC. Vulnerability and Responses to Risks in Rural India. Ssrn. 2013.

41. Soliman AA, Abdulrahman BM., Ali J. Water associated diseases amongst children in IDPs camps and their relation to family economics status: case study of Abu Shock IDPs camp, North Darfur State, Sudan. 2017;5(201 7).

42. Feikin DR, Nguyen LM, Adazu K, Ombok M, Audi A, Slutsker L, et al. The impact of distance of residence from a peripheral health facility on pediatric health utilisation in rural western Kenya. Trop Med Int Heal. 2009;14(1):54-61.

43. Cançado V, Brasil L, Nascimento N, Guerra A. Flood risk assessment in an urban area: Measuring hazard and vulnerability. In: 11th International Conference on Urban Drainage, Edinburgh, Scotland, UK, 2008 Flood. 2008. p. 1-10.

44. Benage M, Gregg Greenough P, Vinck P, Omeira N, Pham P. An assessment of antenatal care among Syrian refugees in Lebanon. Confl Health. 2015;9(1):1-11.

45. Sphere Project. The Sphere Handbook: Humanitarian Charter and Minimum Standards in Humanitarian Response [Internet]. Vol. 1, CHS Alliance, Sphere Association and Groupe URD. 2018. 402 p. Available from: www.practicalactionpublishing.org/sphere

46. Connolly MA, Gayer M, Ryan MJ, Salama P, Spiegel P, Heymann DL. Communicable Diseases in Complex Emergencies. Lancet. 2004;364:1974-83.

47. Jean-Baptiste N, Kuhlicke C, Kunath A, Kabisch S. Review and evaluation of existing vulnerability indicators for assessing climate related vulnerability in Africa. 2011.

\section{Figures}




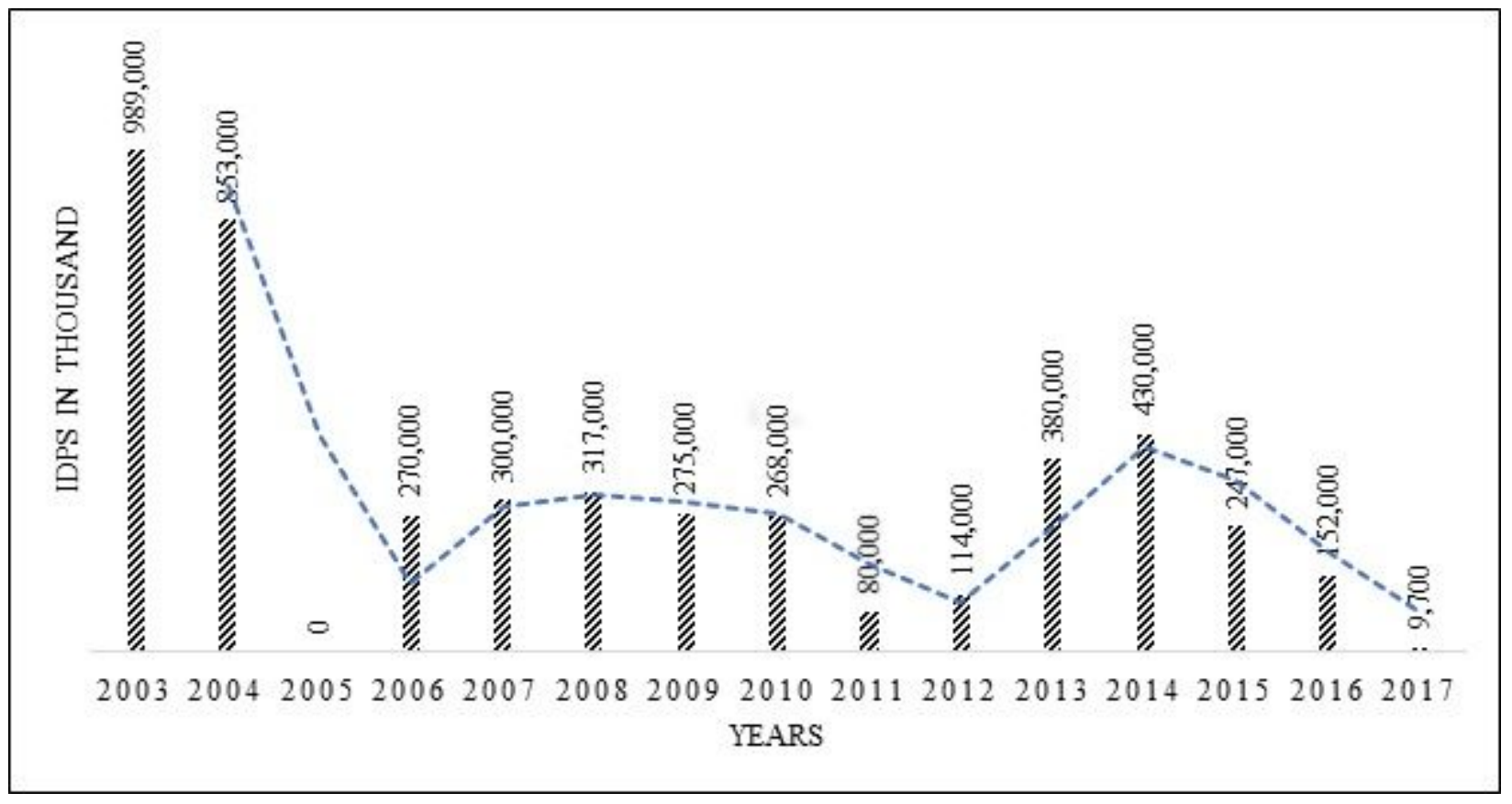

\section{Figure 1}

Number of IDPs displaced by conflict in Darfur (2003 - 2017). Source: (3) 

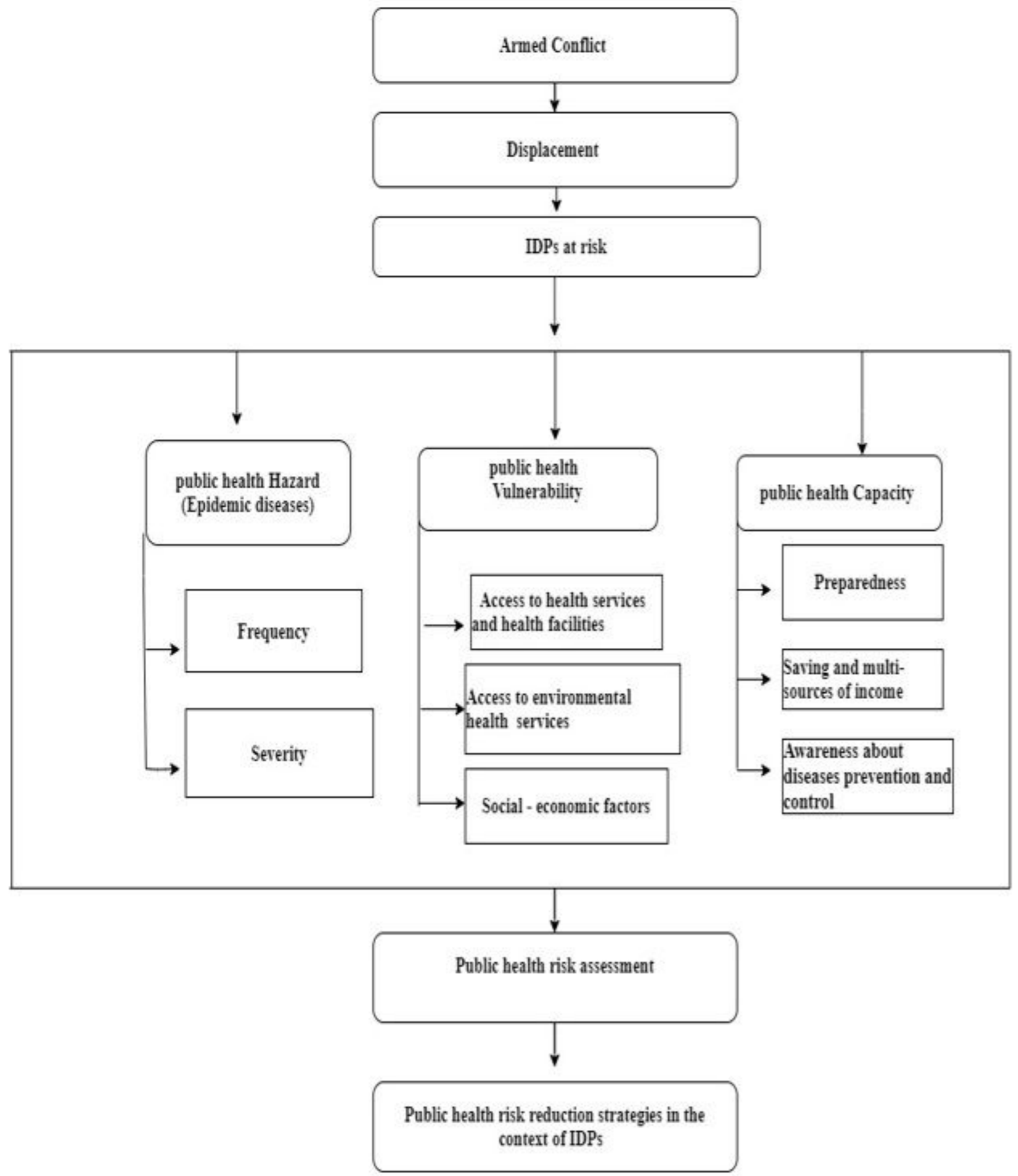

Figure 2

Conceptual Framework (developed by author) 


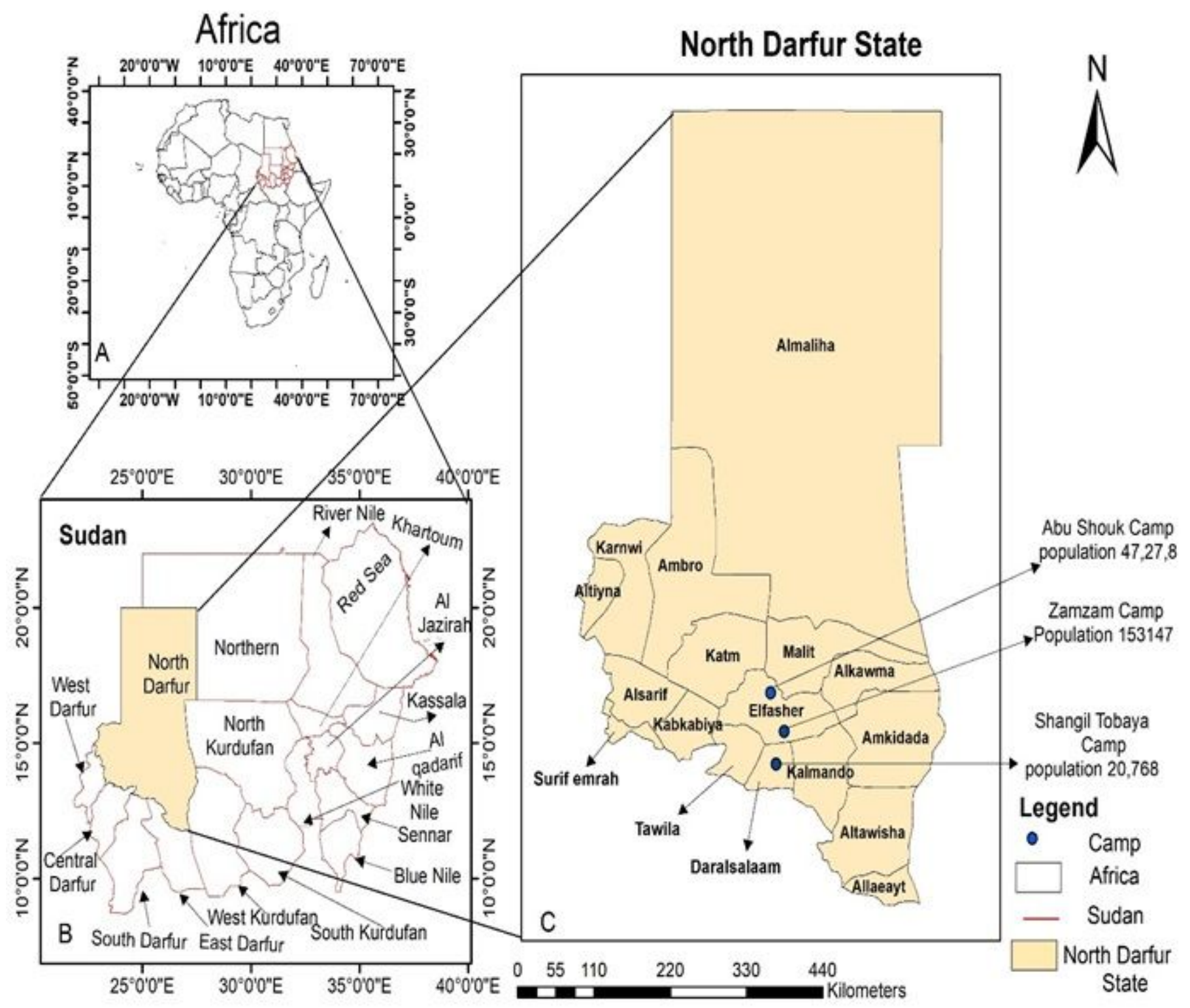

Figure 3

Map of the study area (developed by author) 


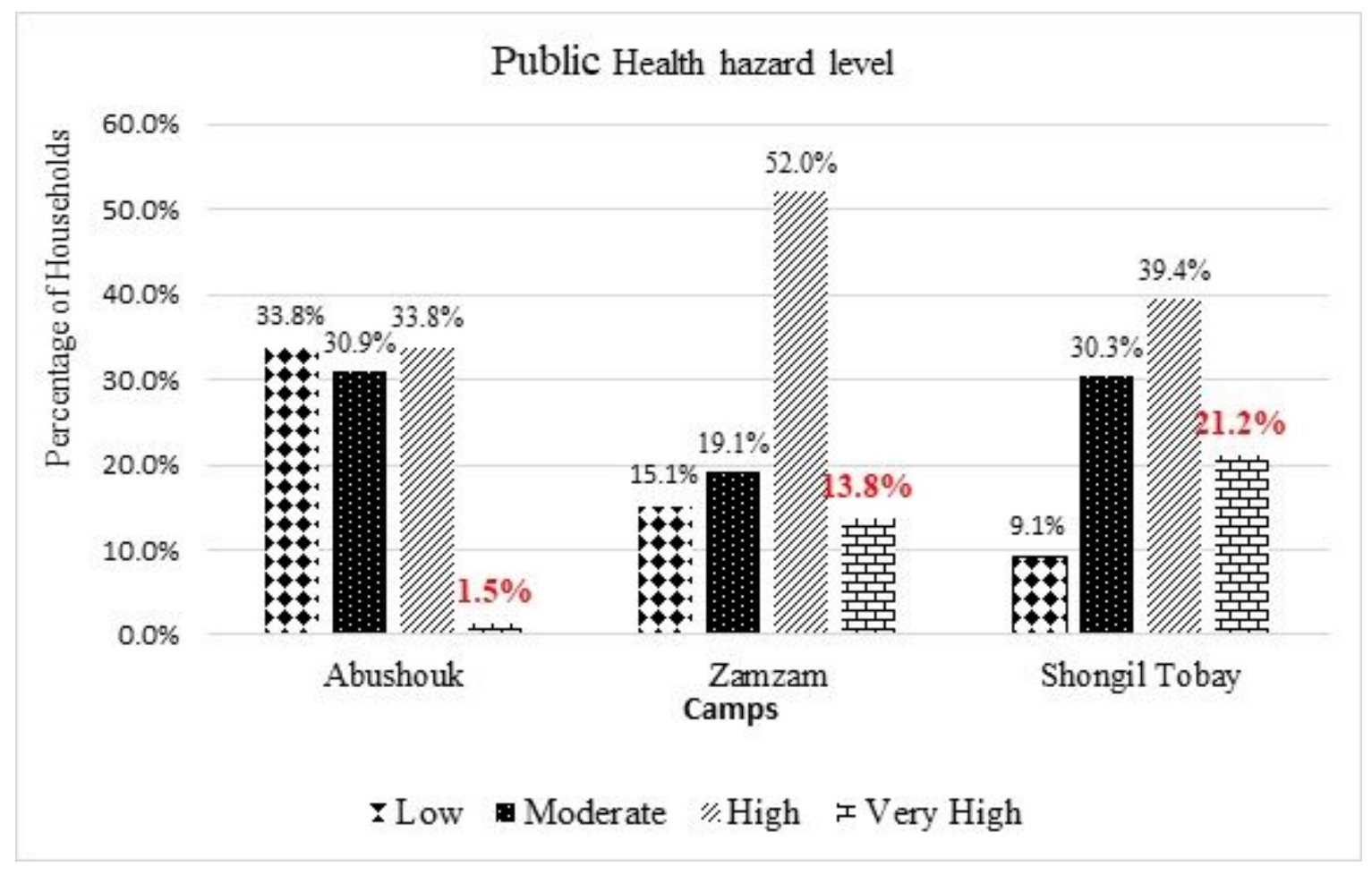

Figure 4

Households health hazard level in three IDP camps

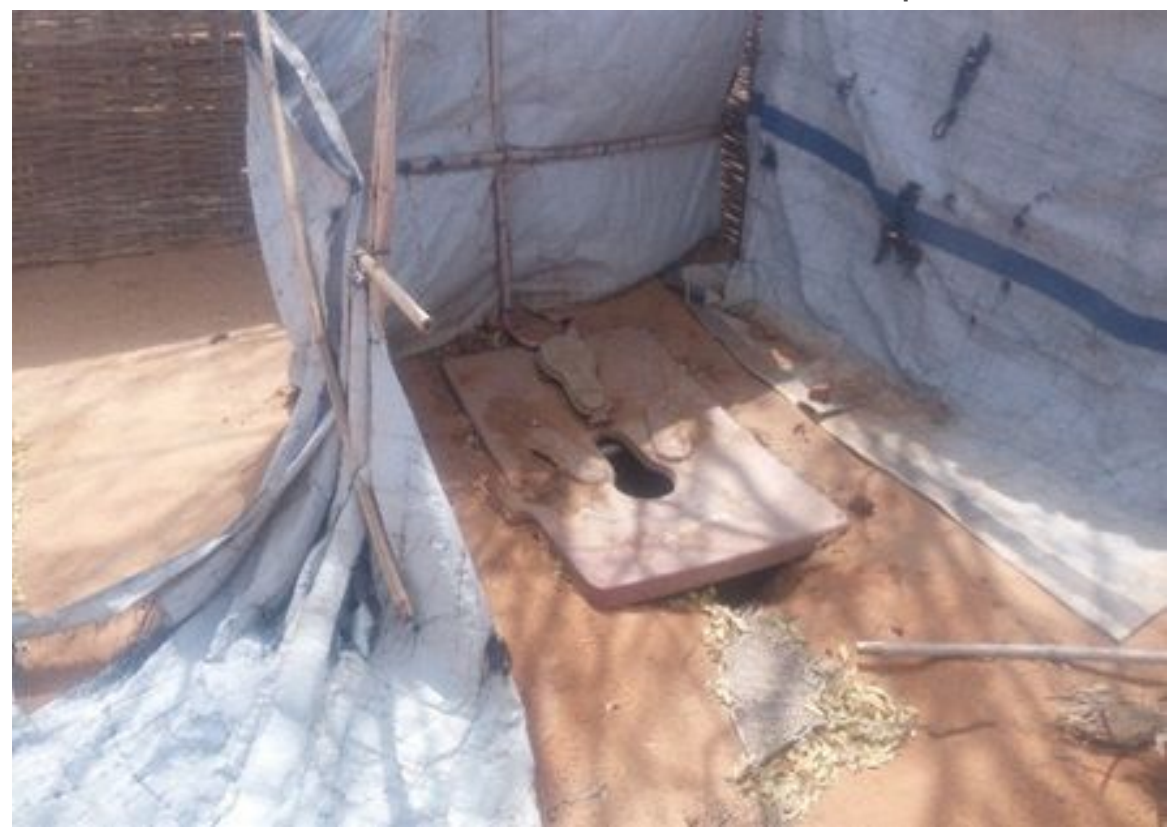

Figure 5

To show that majority of emergency latrines either damaged or full and dirty and thus not convenient for use. (photo taken by author) 


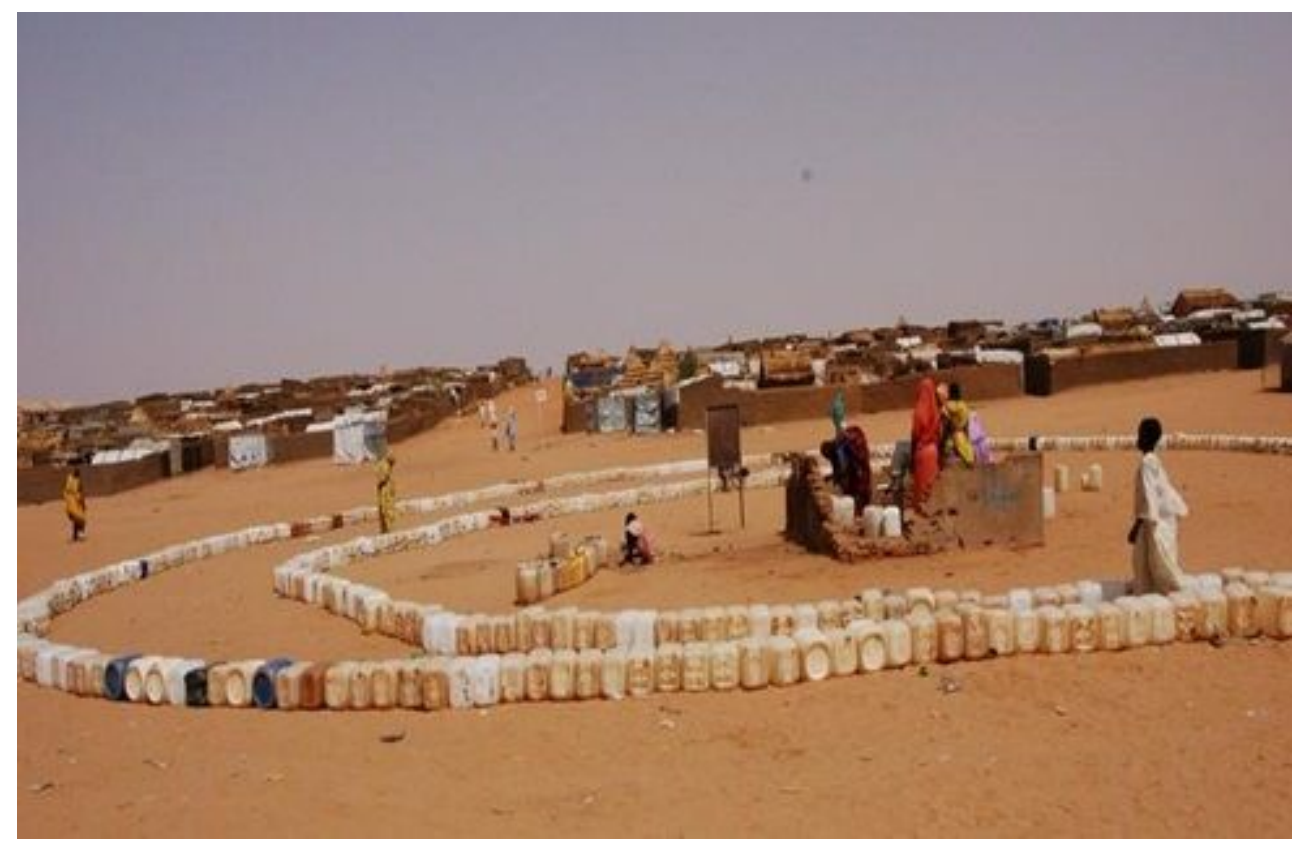

\section{Figure 6}

Water scacrity/ water point in Zamzam camp (photo taken by author)

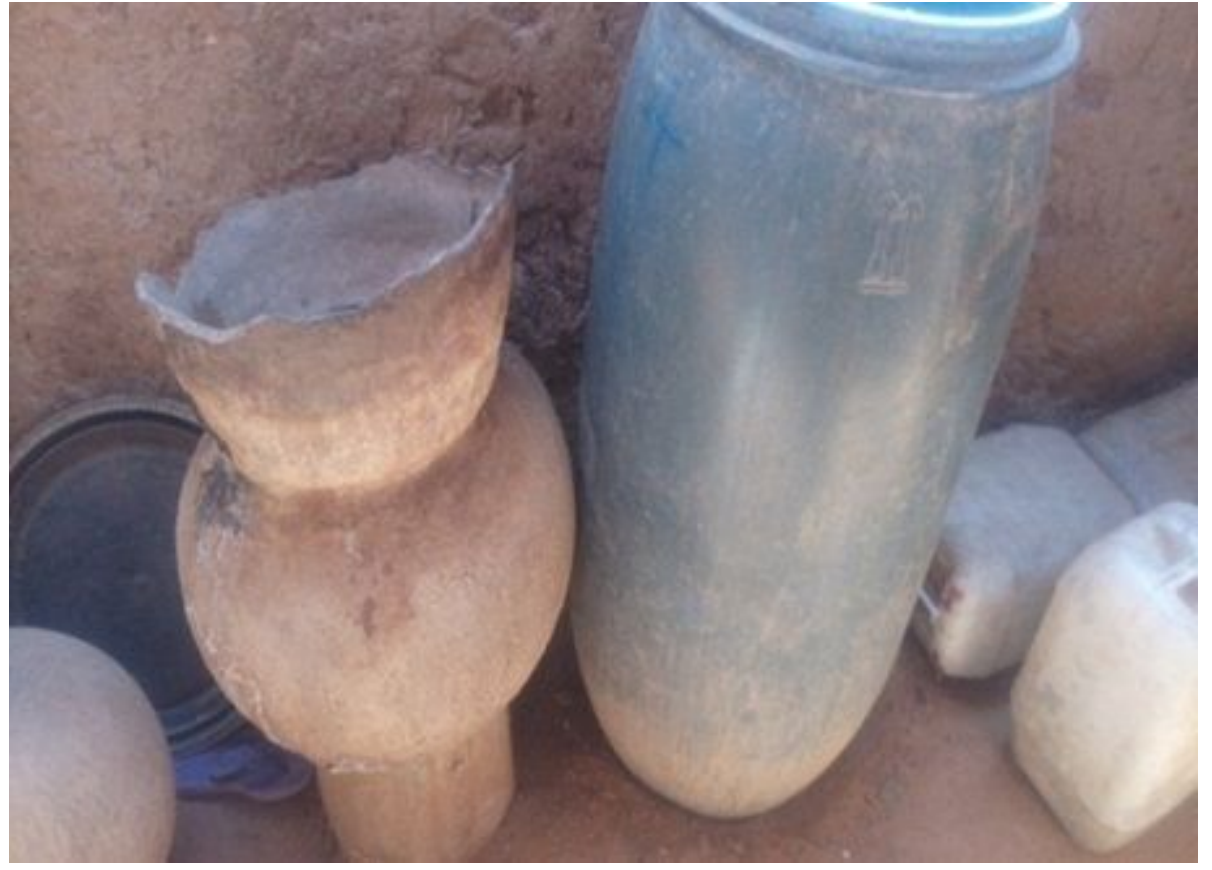

\section{Figure 7}

Type of water storage in Zamzam (photo taken by author) 


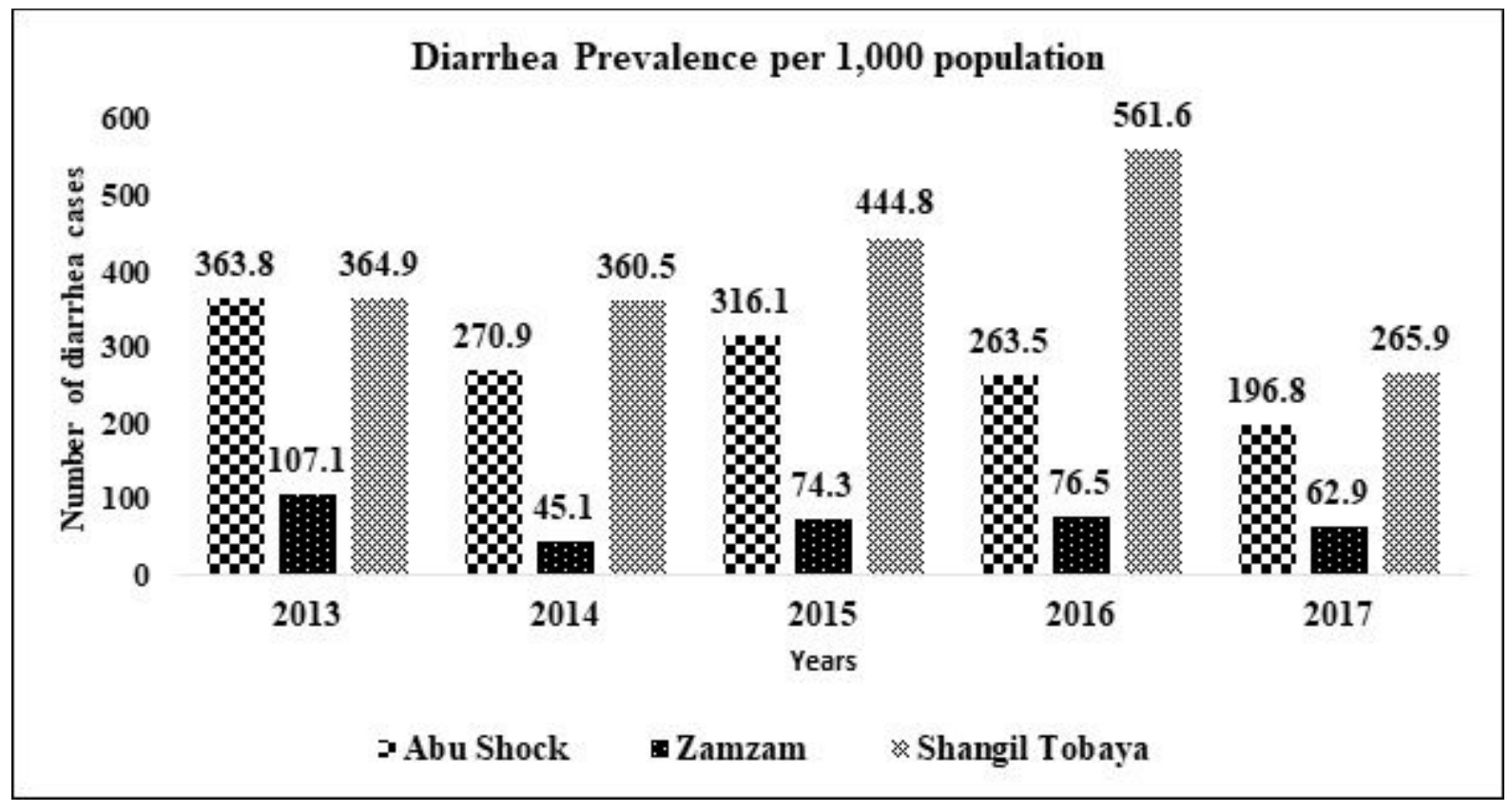

Figure 8

The prevalence rate of diarrhea per 1,000 population in the targeted camps from 2013 to 2017. (32).

- - Abu Shock -- Zamzam - - Shangil Tobaya

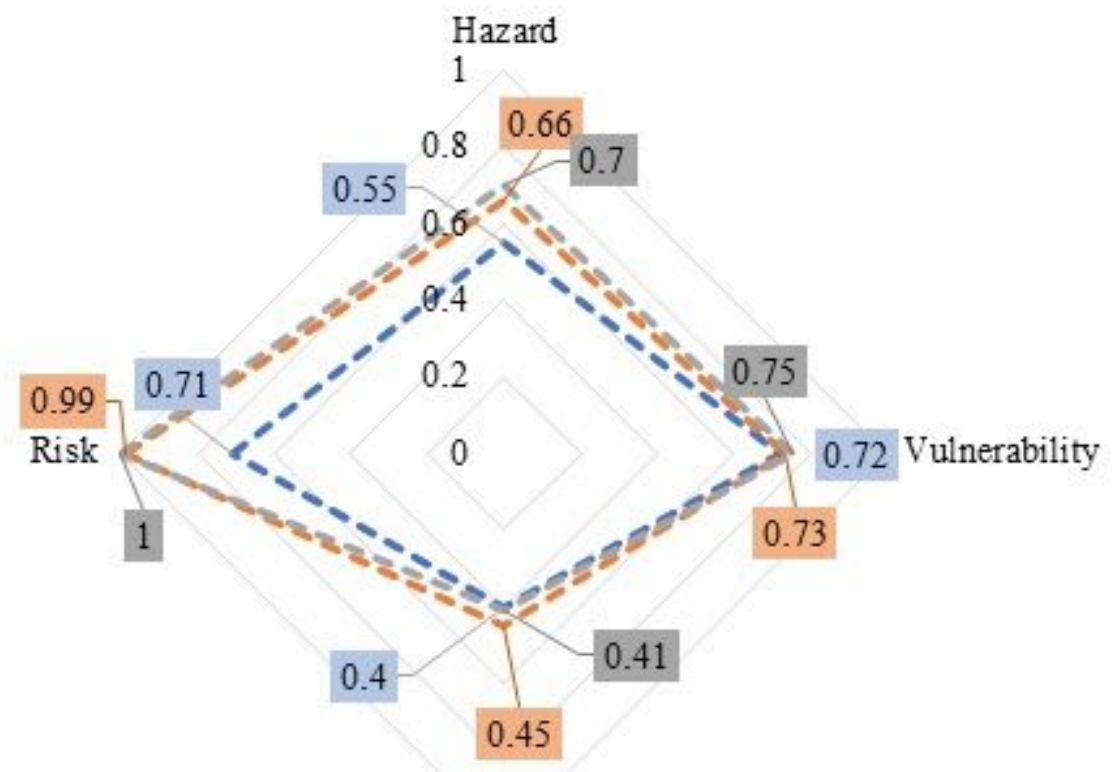

Capacity

Figure 9

Comparative analysis of public health risk components at households' level in three camps. 


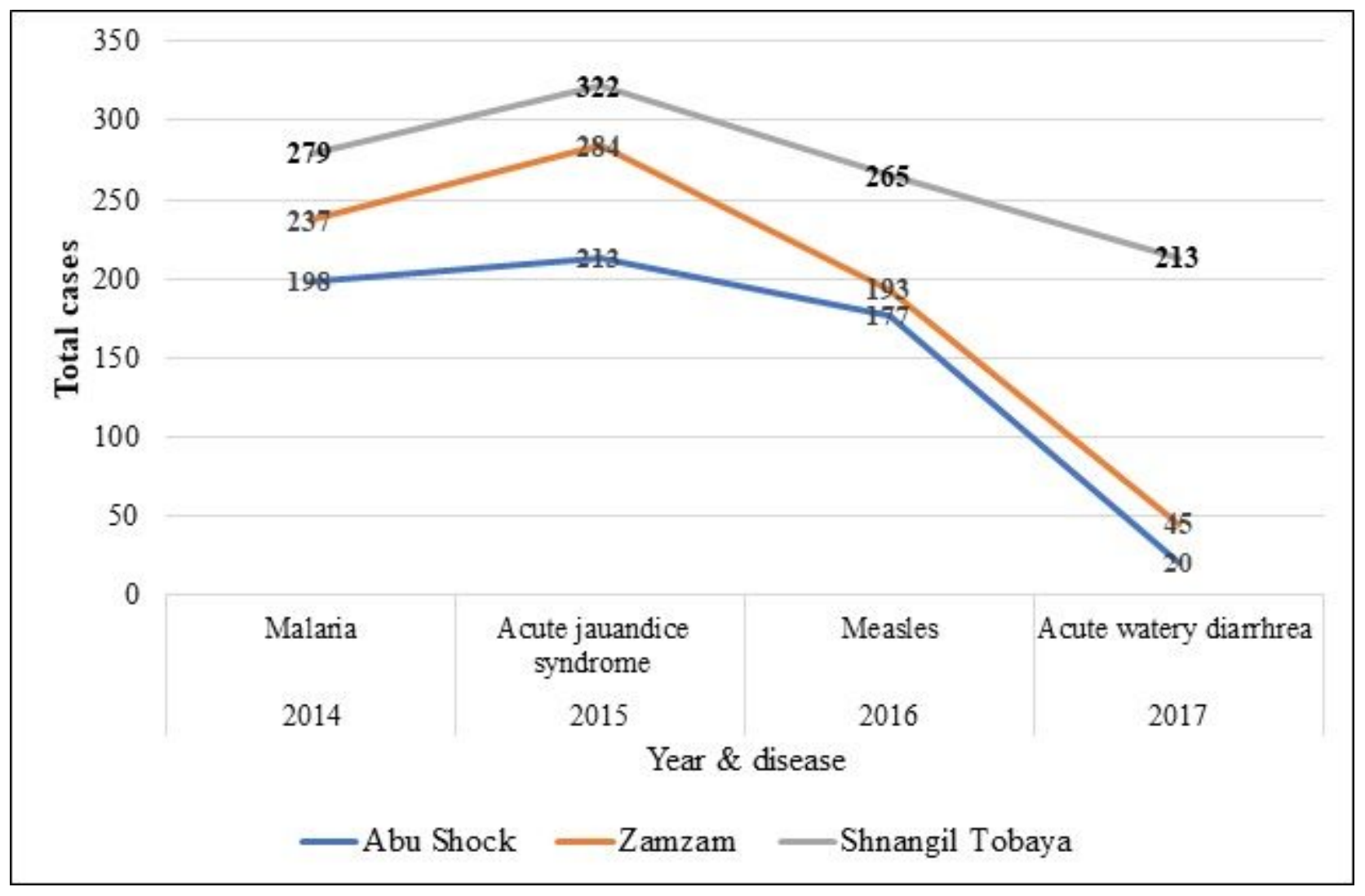

\section{Figure 10}

Diseases outbreaks distribution cases by camp type of diseases and year in North Darfur State, Sudan, 2014-2017. (32) 